\title{
Analysis of risk factors affecting the development of peptic ulcer perforation: case-control study
}

\author{
Sami Akbulut ${ }^{1}$, Ali Riza Caliskan², Hasan Saritas ${ }^{3}$, Khaled Demyati ${ }^{1,4}$, Yilmaz Bilgic ${ }^{2}$, Selver Unsal ${ }^{5}$, \\ Cemalettin Koc ${ }^{1}$, Sezai Yilmaz ${ }^{1}$ \\ 1Department of Surgery and Liver Transplant Institute, Faculty of Medicine, Inonu University, Malatya, Turkey \\ ${ }^{2}$ Department of Gastroenterology, Faculty of Medicine, Inonu University, Malatya, Turkey \\ ${ }^{3}$ Department of Surgical Nursing, Faculty of Health Sciences, Inonu University, Malatya, Turkey \\ ${ }^{4}$ Department of Surgery, An-Najah National University Hospital, An-Najah National University, Nablus, Palestine \\ ${ }^{5}$ Department of Nursing Service, Inonu University, Malatya, Turkey
}

Gastroenterology Rev 2021; 16 (1): 23-28

DOI: https://doi.org/10.5114/pg.2020.94744

Key words: peptic ulcer, perforation, risk factors.

Address for correspondence: Assoc Prof. Sami Akbulut FACS, Department of Surgery and Liver Transplant Institute, Faculty of Medicine, Inonu University, Elazig Yolu 10. Km, Malatya 44280, Turkey, phone: +90 422-3410660, fax: +90 422-3410036, e-mail: akbulutsami@gmail.com

\begin{abstract}
Aim: The aim of the to determine the risk factors associated with increased risk of peptic ulcer perforation (PUP).

Material and methods: The demographic, clinic, and biochemical parameters of 65 patients (PUP group) who underwent PUP surgery at our clinic between June 2009 and September 2016 were compared with the data of 134 patients (control group) who underwent endoscopy at a gastroenterology clinic for dyspeptic complaints. The control group were matched at random in a $1: 2$ ratio with the PUP group. Univariate analyses were used to compare different variables and variables with clinical significance, and $p \leq 0.05$ was used in the backward stepwise logistic regression model.

Results: This study included 65 patients with peptic ulcer perforation aged 17 to 92 years (PUP group) and 134 patients with dyspeptic complaints aged 18 to 87 years (control group). Univariate analysis showed that statistically significant differences were found between groups in terms of non-steroidal anti-inflammatory drugs usage ( $p=0.042$; OR $=1.868)$, smoking $(p<0.001 ; \mathrm{OR}=5.124)$, old age $(p=0.003)$, low body mass index $(\mathrm{BMI})(p<0.001)$, and low hemoglobin $(\mathrm{Hb})(p=0.002)$. However multivariate analysis showed that increasing age $(p=0.004 ; O R=1.035)$, smoking $(p=0.007 ; O R=3.591)$, decreasing $\mathrm{Hb}(p=0.042 ; \mathrm{OR}=1.277)$, and decreasing $\mathrm{BMI}(p<0.001 ; \mathrm{OR}=1.669)$ were independent clinically significant risk factors for development of PUP.

Conclusions: This study showed that decreased BMI, decreased $\mathrm{Hb}$, increased age, and smoking were independent risk factors for development of PUP. Thus, this group of patients needs particular attention paid to suggestive symptoms with early diagnosis and optimal management of peptic ulcer disease.
\end{abstract}

\section{Introduction}

Peptic ulceration is a major public health problem. It is estimated that each year, peptic ulcer disease (PUD) affects 4 million people around the world [1]. Individuals with PUD are at risk of developing complications such as gastroduodenal haemorrhage, perforation, penetration, and obstruction, and mortality among patients with these complications is high [2]. Peptic ulcer perforation (PUP) is a frequent emergency condition worldwide associated with high mortality if left untreated. It presents as an acute abdominal condition, with localised or generalised peritonitis and a high risk for developing sepsis and death. PUP is a surgical emergency and carries a mortality ranging from $1.3 \%$ to $20 \%$. Thirty-day mortality rates reaching $20 \%$ and 90 -day mortality rates of up to $30 \%$ have been reported [3-5].

Being closely related to advanced age, increased burden of comorbidity may partially explain the higher mortality among elderly patients; however, several other factors affect this high mortality [6]. While Helicobacter pylori and use of non-steroidal anti-inflammatory drugs (NSAID) are frequent causes of PUP, demographic differences in age, gender, perforation location, and aetiology exist between countries, as do mortality 
rates, with several risk factors potentially influencing the development of PUP and postoperative mortality.

\begin{abstract}
Aim
This study's primary aim was to determine both the risk factors affecting PUP and the risk factors affecting the mortality in patients who underwent surgery due to PUP. Identifying patients at increased risk with application of optimised management and prophylactic measures is expected to reduce mortality in this group of patients.
\end{abstract}

\section{Material and methods}

Electronic file records of adult patients who underwent surgery with the preliminary diagnosis of PUP at the Department of Surgery, Inonu University Faculty of Medicine between 15 June 2009 and 15 September 2016 were reached. The patient list was obtained by entering the codes "609.710, 609.890, 609.900" into the ENLIL patient information system used in our hospital. A total of 68 patients with PUP, whose demographic (age, gender, height, weight, body mass index (BMI)), clinical (blood group, smoking, alcohol use, diabetes, chronic kidney disease, chronic heart disease, non-steroidal anti-inflammatory drugs (NSAID) use) and biochemical (white blood cell (WBC), mean cell haemoglobin $(\mathrm{MCH})$, mean corpuscular volume (MCV), red cell distribution width (RDW), platelet distribution width (PDW), lymphocyte, neutrophil, PDW, and platelet) parameters were reached, were found suitable for inclusion, and this group was defined as the case group (PUP group). To create a control group, 136 consecutive patients with dyspeptic complaints, who underwent panendoscopy at the Gastroenterology Department of our hospital due to dyspeptic complaints, were selected as the control group. Both groups were compared in terms of the above-mentioned demographic and clinical parameters. All patients were contacted by phone to learn the current status. However, 3 patients in the case group and 2 patients in the control group were excluded because they could not be reached by phone. As a result, 65 patients in the case group and 134 patients in the control group of this study were included in the analysis. After the approval of the Inonu University Institutional Review Board for non-interventional studies (Approval No: 2018/6-4), the patients' files were retrospectively examined.

\section{Statistical analysis}

The statistical analyses were performed using IBM SPSS Statistics v25.0 (Statistical Package for the Social Sciences, Inc., Chicago, IL, USA). The quantitative variables were expressed as mean \pm SD, median, and min- max. The qualitative variables were reported as numbers and percentages (\%). Kolmogorov-Smirnov test was used to assess normality of quantitative variables' distribution. Mann Whitney- $U$ test was used to compare quantitative variables. Pearson's $\chi^{2}$ and Fisher's exact tests were used to compare qualitative variables. Variables such as age, smoking, NSAIDs, haemoglobin $\mathrm{Hb}), \mathrm{BMI}$, height, and weight, which were thought to be both clinically and statistically significant were taken into a backward stepwise (likelihood ratio) logistic regression model to determine independent risk factors for PUP. Hosmer-Lemeshow test was used for the goodness of fit of logistic regression models. $P \leq 0.05$ was considered statistically significant. This retrospective study was approved by the Inonu University Rectorate Ethics Committee (Approval No. 2018/6-4)

\section{Results}

This study included 65 patients with peptic ulcer perforation aged 17 to 92 years (PUP group) and 134 patients with dyspeptic complaints aged 18 to 87 years (control group). A statistically significant difference was found between the groups in terms of age $(p=0.003)$, height $(p<0001)$, weight $(p<0001)$, BMI $(p<0001)$, $\mathrm{Hb}(p=0.002)$, WBC $(p<0001)$, neutrophil $(p<0001)$, lymphocyte $(p<0001), \operatorname{MPV}(p<0001)$, RDW $(p<$ $0.001)$, PDW $(p<0001)$, platelets $(p=0.016)$, NSAID usage $(p=0.042, \mathrm{OR}=1.868)$, and smoking $(p<0001$, $\mathrm{OR}=5.124)$ variables. However, no statistically significant difference was found between the groups in terms of gender $(p=0.070)$, blood groups $(p=0.509)$, diabetes mellitus $(p=0.316)$, alcohol intake $(p=0.352)$, cardiovascular disease $(p=0.290)$, renal disease $(p=0.446)$, and pulmonary disease $(p=0.215)$ variables (Tables I, II). To demonstrate whether the variables were risk factors for PUP development, seven clinical significant variables (age, smoking, NSAIDs, $\mathrm{Hb}, \mathrm{BMI}$, height, and weight), with $p \leq 0.05$, were taken into the logistic regression model, and this model showed that increasing age $(p=0.004 ; \mathrm{OR}=1.035)$, smoking $(p=0.007$; $\mathrm{OR}=3.591)$, decreasing $\mathrm{Hb}(p=0.042 ; \mathrm{OR}=1.277)$, and decreasing BMI $(p<0.001$; OR = 1.669) were independent risk factors for development of PUP (Table III).

\section{Discussion}

A PUD is a defect in the gastric or duodenal wall that extends through the muscularis mucosa into the deeper layers of the wall. PUD remains a major public health problem worldwide, and a recent systemic review showed that the 1-year prevalence of PUD based on physician diagnosis ranged from $0.12 \%$ to $1.50 \%$, and that based on hospitalisation data ranged from $0.10 \%$ to $0.19 \%[7]$. 
PUD can be complicated by haemorrhage, perforation, penetration, or obstruction. These complications represent the most common indications for surgery in PUD. Although there has been a sharp decline in elective surgery for PUD, the rates of emergency surgery for complicated PUD have been stable over time. Patients with gastric ulcers are also at risk of developing gastric malignancy. PUP typically presents with sudden onset of severe abdominal pain but may be less dramatic, particularly in hospitalised, elderly, and immunocompromised patients. The resulting peritonitis is often generalised but can be localised when the perforation is walled off by adjacent viscera and structure. In general, all patients with PUP require prompt resuscitation, intravenous antibiotics, analgesia, proton pump inhibitory medications, nasogastric tube, urinary catheter, and surgical source control. Perforated gastric ulcers are best treated by simple wedge resection to eliminate the perforation and exclude malignancy. If wedge resection of the ulcer cannot be performed due to ulcer location in the juxtapyloric region, multiple biopsies of the ulcer are taken and omental patching is performed. Perforated duodenal ulcers are best managed by simple omental patching and peritoneal debridement followed by Helicobacter pylori eradication. An acid-reducing procedure, which is usually not performed in emergency surgeries for PUP, should be considered in stable patients who have failed medical therapy.

PUP carries a mortality ranging from $1.3 \%$ to $20 \%$. Thirty-day mortality rates reaching $20 \%$ and 90 -day mortality rates of up to $30 \%$ have been reported [3-5]. Studies showed that while haemorrhage remained the most common complication, perforation had the highest mortality. PUP had a five-fold higher mortality rate than bleeding ulcers and was the single most important contributor to inpatient mortality [8]. While there has been a significant decrease in PUD mortality with a significant increase in the use of therapeutic endoscopy for bleeding ulcer [8], mortality rates for PUP have remained stable, despite progress in perioperative care, imaging techniques, and surgical management [3]. In the present study the 30 -day mortality rate is $12.3 \%$; the results are consistent with the literature.

Despite continuous exposure to several noxious factors, under normal conditions the gastric mucosa is able to maintain structural integrity and function. However, gastric mucosal injuries may occur when harmful factors overcome an intact mucosal defence or when the mucosal defensive mechanisms are impaired. Mucosal injury and, thus, peptic ulcer occur when the balance between the aggressive noxious factors and the defensive mechanisms is disrupted [9].

Aggressive noxious agents may include injurious factors of endogenous origin such as reflux of alkaline
Table I. Comparison of PUP and Control groups in terms of continuous variables

$\begin{array}{lcc}\text { Parameter } & \begin{array}{c}\text { PUP group } \\ (n=65)\end{array} & \begin{array}{c}\text { Control group } \\ (n=134)\end{array}\end{array}$

Height: $<0.001$

\begin{tabular}{lcc}
\hline Mean \pm SD & $172.5 \pm 5.0$ & $168.6 \pm 8.1$ \\
\hline Median (min.-max.) & $174(162-186)$ & $170(150-190)$ \\
\hline
\end{tabular}

\begin{tabular}{|c|c|c|c|}
\hline Weight: & & & $<0.001$ \\
\hline Mean \pm SD & $62.2 \pm 7.3$ & $76.0 \pm 12.7$ & \\
\hline Median (min.-max.) & $62(48-82)$ & $75(47-117)$ & \\
\hline $\mathrm{Hb}:$ & & & 0.002 \\
\hline Mean \pm SD & $13.5 \pm 2.4$ & $14.5 \pm 1.9$ & \\
\hline Median (min.-max.) & $13.8(7.9-18.5)$ & $14.6(7.9-17.9)$ & \\
\hline
\end{tabular}

\begin{tabular}{|c|c|c|c|}
\hline WBC: & & & $<0.001$ \\
\hline Mean \pm SD & $11.9 \pm 7.2$ & $7.9 \pm 1.9$ & \\
\hline Median (min.-max.) & $10.9(1.1-36.9)$ & $7.7(4.4-13.3)$ & \\
\hline Neutrophil: & & & $<0.001$ \\
\hline Mean \pm SD & $9.7 \pm 6.9$ & $4.5 \pm 1.5$ & \\
\hline Median (min.-max.) & $8.3(0.1-35.4)$ & $4.3(1.9-9.7)$ & \\
\hline
\end{tabular}

\begin{tabular}{|c|c|c|c|}
\hline \multicolumn{3}{|l|}{ Lymphocyte: } & \multirow[t]{3}{*}{$<0.001$} \\
\hline Mean \pm SD & $0.97 \pm 0.6$ & $2.5 \pm 0.1$ & \\
\hline Median (min.-max.) & $0.8(0.0-2.7)$ & $2.5(0.8-5.1)$ & \\
\hline \multicolumn{3}{|l|}{ Platelets: } & \multirow[t]{3}{*}{0.016} \\
\hline Mean \pm SD & $253.6 \pm 127$ & $267.6 \pm 65.2$ & \\
\hline Median (min.-max.) & $240(48-731)$ & $259(21-453)$ & \\
\hline \multicolumn{3}{|l|}{ MPV: } & \multirow[t]{3}{*}{$<0.001$} \\
\hline Mean \pm SD & $8.76 \pm 1.5$ & $10.3 \pm 0.73$ & \\
\hline Median (min.-max.) & $8.5(6.3-13.5)$ & $10.2(8.4-12.5)$ & \\
\hline \multicolumn{3}{|l|}{ RDW: } & \multirow[t]{3}{*}{$<0.001$} \\
\hline Mean \pm SD & $15.3 \pm 3.1$ & $13.7 \pm 1.6$ & \\
\hline Median (min.-max.) & $14.4(12.1-27.7)$ & $13.4(11.2-20.9)$ & \\
\hline \multicolumn{3}{|l|}{ PDW: } & \multirow[t]{3}{*}{$<0.001$} \\
\hline Mean \pm SD & $16.2 \pm 2.2$ & $12.1 \pm 1.47$ & \\
\hline Median (min.-max.) & $16.7(10.9-24.3)$ & $11.8(8.3-16.2)$ & \\
\hline
\end{tabular}


Table II. Comparison of PUP and Control groups in terms of categorical variables

\begin{tabular}{|c|c|c|c|c|c|}
\hline Parameter & PUP group $(n=65)$ & Control group $(n=134)$ & $P$-value & OR & $95 \% \mathrm{Cl}$ \\
\hline Gender: & & & 0.070 & & \\
\hline Male & $50(76.9)$ & $86(63.2)$ & & & \\
\hline Female & $15(23.1)$ & $48(35.8)$ & & & \\
\hline NSAID: & & & 0.042 & 1.868 & $1.018-3.429$ \\
\hline Yes & $41(63.1)$ & $64(47.8)$ & & & \\
\hline No & $24(36.9)$ & $70(52.2)$ & & & \\
\hline Smoking: & & & $<0.001$ & 5.124 & $2.707-9.701$ \\
\hline Yes & $43(66.2)$ & $37(27.6)$ & & & \\
\hline No & $22(33.8)$ & $97(72.4)$ & & & \\
\hline Blood groups: & & & 0.509 & & \\
\hline $\mathrm{ORh}(+)$ & $24(58.5)$ & $63(47.0)$ & & & \\
\hline $\mathrm{A} \operatorname{Rh}(+)$ & $8(19.5)$ & $47(35.1)$ & & & \\
\hline $\mathrm{BRh}(+)$ & $8(19.5)$ & $8(19.5)$ & & & \\
\hline $\mathrm{AB} \operatorname{Rh}(+)$ & $1(2.4)$ & $1(2.4)$ & & & \\
\hline Diabetes mellitus: & & & 0.316 & & \\
\hline Yes & $10(15.4)$ & $14(10.4)$ & & & \\
\hline No & $55(84.6)$ & $120(89.6)$ & & & \\
\hline Alcohol: & & & 0.352 & & \\
\hline Yes & $5(7.7)$ & $6(4.5)$ & & & \\
\hline No & $60(92.3)$ & $128(95.5)$ & & & \\
\hline Cardiovascular disease & & & 0.290 & & \\
\hline Yes & $7(10.8)$ & $22(16.4)$ & & & \\
\hline No & $58(89.2)$ & $112(83.6)$ & & & \\
\hline Renal disease: & & & 0.446 & & \\
\hline Yes & $3(4.6)$ & $10(7.5)$ & & & \\
\hline No & $62(95.4)$ & $124(92.5)$ & & & \\
\hline Pulmonary disease: & & & 0.215 & & \\
\hline Yes & $9(13.8)$ & $11(8.2)$ & & & \\
\hline No & $56(86.2)$ & 123 (91.8) & & & \\
\hline
\end{tabular}

Table III. Determination of factors affecting PUP using backward stepwise logistic regression model

\begin{tabular}{|c|c|c|c|c|c|c|c|}
\hline \multirow[t]{2}{*}{ Parameter } & \multirow[t]{2}{*}{ B } & \multirow[t]{2}{*}{ SE } & \multirow[t]{2}{*}{ Wald } & \multirow[t]{2}{*}{ Sig. } & \multirow[t]{2}{*}{$\operatorname{Exp}(B)$} & \multicolumn{2}{|c|}{$95 \% \mathrm{Cl}$ for $\operatorname{EXP}(\mathrm{B})$} \\
\hline & & & & & & Lower & Upper \\
\hline Age & 0.034 & 0.012 & 8.414 & 0.004 & 1.035 & 1.011 & 1.059 \\
\hline Smoking & 1.278 & 0.474 & 7.268 & 0.007 & 3.591 & 1.418 & 9.096 \\
\hline NSAIDs & 0.756 & 0.463 & 2.658 & 0.103 & 2.159 & 0.858 & 5.280 \\
\hline $\mathrm{Hb}$ & -0.244 & 0.120 & 4.115 & 0.042 & 0.783 & 0.619 & 0.992 \\
\hline BMI & -0.512 & 0.085 & 36.227 & 0.000 & 0.599 & 0.507 & 0.708 \\
\hline Constant & 12.919 & 2.711 & 22.710 & 0.000 & & & \\
\hline
\end{tabular}


duodenal contents containing bile and pancreatic enzymes. Alcohol, cigarette smoking, drugs - particularly aspirin and aspirin-like drugs, and steroids are among exogenous mucosal irritants that can inflict mucosal injury [9]. It can alter the mucosal defence by allowing back diffusion of hydrogen ions and subsequent epithelial cell injury. The defensive mechanisms include tight intercellular junctions, mucus, bicarbonate, mucosal blood flow, cellular restitution, and epithelial renewal. PUD occurs mainly in the stomach (gastric ulcer) or proximal duodenum (duodenal ulcer). It was also shown that duodenal ulcers occur in gastric metaplasia mucosa in the duodenum [10].

It is unclear why some patients perforate and others do not. It might be related to the presence of continuous insult exposure without adequate management. Furthermore, there are geographical differences in the aetiology and variation in risk factors for PUP [3]. Only about a third of patients with PUP have a known history of peptic ulcer at the time of diagnosis. Furthermore, some patients develop very small $(<5 \mathrm{~mm})$ perforations without large mucosal defects, which suggests that the ulcer size is unrelated to perforation risk, while other patients may develop large mucosal defects with perforation of several centimetres.

The most common causes of PUD are H. pylori infection and use of NSAIDs. We were unable to accurately identify the status of $H$. pylori infection in patients in this study due to the retrospective nature of the study. However other studies showed a variable prevalence of H. pylori (0-90\%) in perforated ulcers, and ulcers may also develop in the absence of $H$. pylori infection and NSAID use $[3,11]$.

Most patients with PUD are treated successfully with cure of $H$. pylori infection and avoidance of NSAIDs, along with the appropriate use of antisecretory therapy. When $H$. pylorus colonises the gastric mucosa, inflammation usually results. The causal association between H. pylori gastritis and duodenal ulceration is now well established in the adult and paediatric literature. In patients infected with $H$. pylori, high levels of gastrin and pepsinogen and reduced levels of somatostatin have been measured. In infected patients, exposure of the duodenum to acid is increased. Virulence factors produced by $H$. pylori, including urease, catalase, vacuolating cytotoxin, and lipopolysaccharide, are well described.

Patients with PUD should be tested for infection with $\mathrm{H}$. pylori and treated accordingly [12]. Eradication of $H$. pylori in patients with PUD is associated with higher healing rates in patients with duodenal and gastric ulcers. A meta-analysis of 24 randomised trials showed that the 12-month ulcer remission rates for gastric and duodenal ulcers were significantly higher in patients successfully eradicated of $H$. pylori infection as compared with those with a persistent infection [13]. Furthermore, eradication of $H$. pylori infection is associated with lower recurrence rates in patients with ulcers, who are not placed on maintenance antisecretory therapy [14].

Decreased $\mathrm{BMI}$ and increased age are found to be independent risk factors for the development of PUP on multivariate analysis in this study. There are conflicting data about the association between obesity and risk of PUD $[15,16]$. However, to our knowledge the association between low BMI and increased risk of PUP has not been described before. Further studies are needed to confirm these results. Old age was also found in other studies to be associated with increased risk of PUP and increased mortality in PUP [6, 17]. The proportions of patients with comorbidities and taking NSAIDs were reported to be significantly higher in the old age group; a higher proportion of patients in the old age group had PUP over $1 \mathrm{~cm}$ in size [17].

NSAIDs are one of the most widely used therapeutic agents, both prescribed and over the counter. NSAIDs are a broad class of non-glucocorticoid drugs, which are extensively used in anti-inflammatory, analgesic, and antipyretic therapies. However, NSAIDs are a leading cause of drug-related morbidity, especially in the elderly and patients with comorbidities. It may cause many side effects, most commonly in the gastrointestinal (GI) tract, cardiovascular system, kidney, liver, central nervous system, and haematopoietic system. About a quarter of chronic NSAID users will develop PUD, and 2-4\% will bleed or perforate. In NSAID users the size of the PUP was described as larger than in other groups, and hospital stays were relatively longer than for the H. pylori infected group, although statistically insignificant [17-19]. Proton-pump inhibitors have been proven efficacious in healing NSAID-associated ulcers because they provide potent and long-lasting inhibition of gastric acid secretion. Co-administration of NSAIDs and proton-pump inhibitors has been used to decrease upper-GI tract adverse events [19]. In the present study NSAID use was shown to increase PUP risk 1.8 fold with univariate analysis, but the results could not be confirmed with multivariate analysis. One of the limiting factors of the present study is that the patients with PUP were not given adequate information about the size of PUP in the operation report. Therefore, we cannot comment on whether the size of PUP of patients using NSAIDs are different from others.

Smoking and chronic nicotine treatment play a role in the pathogenesis of PUD in several ways. They stimulate basal acid output, which is more pronounced in smokers having duodenal ulcers, by increasing hista- 
mine release and pepsinogen secretion. Long-term nicotine treatment in rats also significantly decreases total mucus neck cell population and neck-cell mucus volume. Smoking also increases the bile salt reflux rate and gastric bile salt concentration. Nicotine not only induces ulceration, but also potentiates ulceration caused by $H$. pylori, alcohol, nonsteroidal anti-inflammatory drugs, or cold restrain stress [20]. Variable results are reported with regard to its clinical significance and association with PUP in the literature [17, 18, 21]. Multivariate analysis in the present study showed that smoking $(p=0.007$; OR $=3.591)$ is an independent risk factor for development of PUP.

\section{Conclusions}

PUP is a serious disease complication. This study showed that decreased $\mathrm{BMI}$, decreased $\mathrm{Hb}$, increased age, and smoking were independent risk factors for development of PUP. This group of patients needs particular attention regarding suggestive symptoms with early diagnosis and optimal management of their PUD.

\section{Conflict of interest}

The authors declare no conflict of interest.

\section{References}

1. Zelickson MS, Bronder CM, Johnson BL, et al. Helicobacter pylori is not the predominant etiology for peptic ulcers requiring operation. Am Surg 2011; 77: 1054-60.

2. Lau JY, Sung J, Hill C, et al. Systematic review of the epidemiology of complicated peptic ulcer: incidence, recurrence, risk factors and mortality. Digestion 2011; 84: 102-13.

3. Soreide K, Thorsen K, Harrison EM, et al. Perforated peptic ulcer. Lancet 2015; 386: 1288-98.

4. Daniel VT, Wiseman JT, Flahive J, et al. Predictors of mortality in the elderly after open repair for perforated peptic ulcer disease. J Surg Res 2017; 215: 108-13.

5. Soreide K, Thorsen K, Soreide JA. Strategies to improve the outcome of emergency surgery for perforated peptic ulcer. Br J Surg 2014; 101: e51-64.

6. Unver M, Firat O, Unalp OV, et al. Prognostic factors in peptic ulcer perforations: a retrospective 14-year study. Int Surg 2015; 100: 942-8.

7. Sung JJ, Kuipers EJ, El-Serag HB. Systematic review: the global incidence and prevalence of peptic ulcer disease. Aliment Pharmacol Ther 2009; 29: 938-46.

8. Wang YR, Richter JE, Dempsey DT. Trends and outcomes of hospitalizations for peptic ulcer disease in the United States, 1993 to 2006. Ann Surg 2010; 251: 51-8.

9. Abdel-Salam OM, Czimmer J, Debreceni A, et al. Gastric mucosal integrity: gastric mucosal blood flow and microcirculation. An overview. J Physiol 2001; 95: 105-27.

10. Calam J, Baron JH. Pathophysiology of duodenal and gastric ulcer and gastric cancer. BMJ 2001; 323: 980-2.
11. Malfertheiner P, Chan FK, McColl KE. Peptic ulcer disease. Lancet 2009; 374: 1449-61.

12. Malfertheiner P, Megraud F, O'Morain CA, et al. Management of Helicobacter pylori infection-the Maastricht V/Florence Consensus Report. Gut 2017; 66: 6-30.

13. Leodolter A, Kulig M, Brasch $\mathrm{H}$, et al. A meta-analysis comparing eradication, healing and relapse rates in patients with Helicobacter pylori-associated gastric or duodenal ulcer. Aliment Pharmacol Ther 2001; 15: 1949-58.

14. Ford AC, Delaney BC, Forman D, et al. Eradication therapy in Helicobacter pylori positive peptic ulcer disease: systematic review and economic analysis. Am J Gastroenterol 2004; 99: 1833-55.

15. Kim J, Kim KH, Lee BJ. Association of peptic ulcer disease with obesity, nutritional components, and blood parameters in the Korean population. PLoS One 2017; 12: e0183777.

16. Pyo JH, Lee H, Kim JE, et al. Obesity and risk of peptic ulcer disease: a large-scale health check-up cohort study. Nutrients 2019; 11: e1288.

17. Yang YJ, Bang CS, Shin SP, et al. Clinical characteristics of peptic ulcer perforation in Korea. World J Gastroenterol 2017; 23: 2566-74.

18. Yamamoto K, Takahashi O, Arioka H, et al. Evaluation of risk factors for perforated peptic ulcer. BMC Gastroenterol 2018; 18: 28.

19. Gwee KA, Goh V, Lima G, et al. Coprescribing proton-pump inhibitors with nonsteroidal anti-inflammatory drugs: risks versus benefits. J Pain Res 2018; 11: 361-74.

20. Maity P, Biswas K, Roy S, et al. Smoking and the pathogenesis of gastroduodenal ulcer: recent mechanistic update. Mol Cell Biochem 2003; 253: 329-38.

21. Chung KT, Shelat VG. Perforated peptic ulcer - an update. World J Gastrointest Surg 2017; 9: 1-12.

Received: 2.02 .2020

Accepted: 24.02 .2020 